\title{
Size Effects in Submicron NiFe/Ag GMR Devices
}

\author{
S. E. Russek, R. W. Cross, S. C. Sanders, and J. O. Oti \\ Electromagnetic Technology Division, National Institute of Standards and Technology, \\ Boulder, CO 80303-3328
}

\begin{abstract}
We have measured the magnetoresistive response of submicron NiFe/Ag giant magnetoresistive (GMR) devices as a function of current density and field angle. In addition to magnetostatic broadening, we observe large jumps in the magnetoresistive response (Barkhausen jumps) due to domain switching. These effects lead to irregular device-specific magnetoresistive response curves. The large Barkhausen jumps are more pronounced at low current density while at high current densities the response is smoother due to self field stabilization. The detailed structure of the Barkhausen jumps is very sensitive to the angle of the applied magnetic field. These effects are general properties of a wide class of GMR materials that rely on incoherent reversal of many small magnetic domains. We compare the experimental data with a micromagnetic simulation which incorporates a phenomenological GMR transport model. The model qualitatively describes the experimental data and provides insight into the detailed micromagnetic behavior of these films.
\end{abstract}

\section{INTRODUCTION}

Multilayer giant magnetoresistive (GMR) thin-film devices show promise for applications in ultra-high-density magnetic recording heads. For these applications the GMR devices must not only have a large response and a low saturation field but must be able to operate at submicron device sizes. Many of the magnetic multilayer systems, especially those that rely on weak exchange or magnetostatic interactions to achieve antiferromagnetic alignment, have complicated domain structures that lead to large resistance jumps (Barkhausen noise) in small devices when magnetic domains flip [1].

In this paper we describe the magnetoresistive characteristics of $\mathrm{NiFe} / \mathrm{Ag}$ devices with line widths as small as $0.7 \mu \mathrm{m}$ and characterize the Barkhausen noise as a function of device size, field angle, and current density. We compare the experimental data with results from a micromagnetic simulation that incorporates a phenomenological GMR model. The main results of this work are that: (1) large resistance jumps can occur in submicron GMR devices and these jumps are sensitive to current density and field angle, (2) the device response can be improved by going to higher current densities due to self field stabilization, and (3) micromagnetic modeling indicates that there is a complicated domain structure in this class of materials.

Manuscript received October 17, 1994.

Contribution of the U.S. Govemment; not subject to copyright.
$\mathrm{NiFe} / \mathrm{Ag}$ multilayers, developed by Hylton et al. [2] [3], are one of the more promising GMR systems due to their high sensitivity and ease of fabrication. The films require short anneals to develop GMR behavior. The best sensitivities are typically achieved with a $340 \mathrm{C}$ anneal, resulting in $\Delta R / R \sim 5 \%$ and $H_{s} \sim 0.8 \mathrm{kA} / \mathrm{m}(10 \mathrm{Oe})$. The annealing temperature may also be varied to achieve zero magnetostriction [4] or obtain broader response for higher field operation. The mechanism responsible for GMR behavior in these films is thought to arise from the break up of the NiFe layers during annealing. Ag diffuses into the grain boundaries and decouples the exchange interaction between grains. Magnetic charges at the surface of the magnetic regions create a weak antiferromagnetic coupling as described in the model proposed by Slonczewski [5]. While the Slonczewski model describes the basic phenomena, it does not describe a real system in which disorder in the exchange interaction, magnetostatic interaction, and magnetic anisotropies can create a complicated domain structure.

\section{EXPERIMENTAL RESULTS}

The samples were fabricated by sputter deposition onto room-temperature oxidized-silicon substrates. The composition of the NiFe alloy sputter target was $\mathrm{Ni}_{82} \mathrm{Fe}_{18}$. The film structure was similar to that developed by Hylton $e t$ al. [2] and consisted of $\mathrm{Si}_{/} / \mathrm{SiO}_{2}(150 \mathrm{~nm}) / \mathrm{Ta}(4.5$ $\mathrm{nm}) / \mathrm{Ag}\left(\mathrm{t}_{\mathrm{Ag}} / 2\right) / \mathrm{NiFe}(2.0 \mathrm{~nm}) /\left[\mathrm{Ag}\left(\mathrm{t}_{\mathrm{Ag}}\right) / \mathrm{NiFe}(2.0 \mathrm{~nm})\right]_{\mathrm{N}-1} / \mathrm{Ag}$ $\left(t_{\mathrm{Ag}} / 2\right) / \mathrm{Ta}(11.0 \mathrm{~nm})$ where the number $\mathrm{N}$ of $\mathrm{NiFe} / \mathrm{Ag}$ bilayers was varied from 5-9 and $\mathrm{Ag}$ spacer layer thickness $t_{\mathrm{Ag}}$ was varied from $2-5 \mathrm{~nm}$. The films were annealed in a rapid thermal annealer in a $5 \% \mathrm{H}_{2}-\mathrm{Ar}$ ambient at temperatures ranging from $320 \mathrm{C}$ to $400 \mathrm{C}$.

The magnetoresistive response as a function of device size is shown in Fig. 1. All devices had a length to width ratio of 10 and an active region (the region between the gold contacts) of one square. The magnetic field was applied perpendicular to the current density in the plane of the film. The large devices, with line widths greater than $4 \mu \mathrm{m}$, show magnetoresistive characteristics similar to the wafer level response. Small devices, with line widths less than $2 \mu \mathrm{m}$, show magnetostatic broadening of the MR response and structure due to domain switching (Barkhausen jumps). For devices with dimensions of $-0.7 \mu \mathrm{m}$, the saturation fields are $\mathrm{H}_{\mathrm{s}} \sim 24 \mathrm{kA} / \mathrm{m}(300 \mathrm{Oe})$ and Barkhausen jumps as large as 
$30 \%$ of the peak response values can be observed. The magnitude of the Barkhausen jumps are sensitive to field angle and current density.

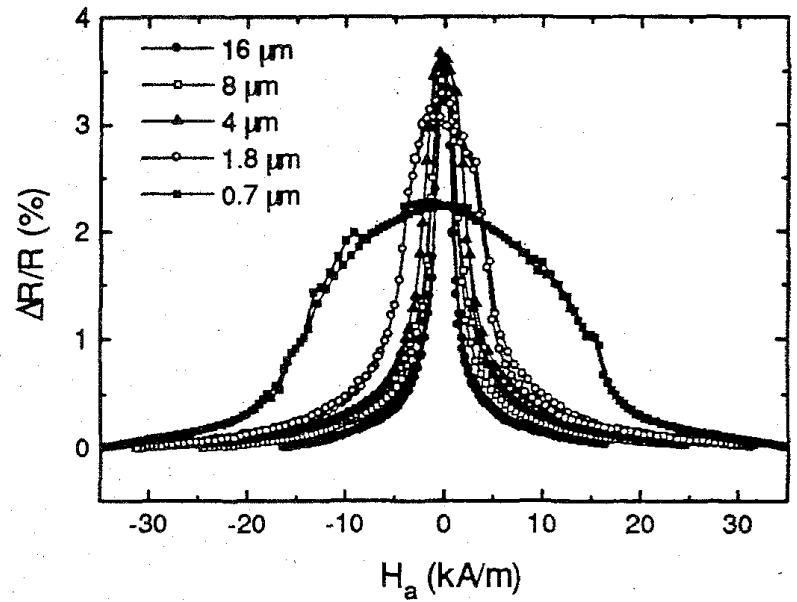

Fig. 1. Magnetoresistive response as a function of device width for a 7 bilayer $\mathrm{NiFe} / \mathrm{Ag}$ film annealed at $340 \mathrm{C}$. The magnetic field was applied perpendicular to the current. The current density was $2.0 \times 10^{5} \mathrm{~A} / \mathrm{cm}^{2}$.

Fig. 2 shows the MR response of a $0.7 \mu \mathrm{m}$ device at current densities of $6.8 \times 10^{5} \mathrm{~A} / \mathrm{cm}^{2}$ to $3.7 \times 10^{7} \mathrm{~A} / \mathrm{cm}^{2}$. In this particular device, the response decreases with increasing current bias and the response becomes smoother. The response amplitude may either increase or decrease with increasing current density depending on the individual device. In all devices, however, the response gets smoother at high current densities. This effect, which we refer to as "self-field stabilization," is due to the current-induced magnetic field. In this type of GMR system, while there is a unique high-field state, there are multiple zero field states, with large antiparallel correlations and similar energies. Switching between these configurations gives rise to the large Barkhausen jumps. The self-field at high current densities causes this multiplicity of states to become energetically unfavorable.

The magnetoresistive response of a $0.7 \mu \mathrm{m}$ device as a function of field angle is shown in Fig. 3. The magnetoresistive response narrows as the field deviates from the perpendicular direction due to magnetostatic effects, and the detailed structure of the Barkhausen noise changes. The response becomes more irregular and large Barkhausen jumps appear. The domain switching structure is a sensitive function of field angle. To replicate a particular curve the field history and the field angle need to be reproduced exactly.

\section{MODELING RESULTS}

We have simulated the magnetoresistive response of NiFe/Ag multilayers using a micromagnetic model which incorporates a simple GMR transport calculation. The model is described in detail by Oti et al. [6]. Briefly, two magnetic layers, separated by a nonmagnetic spacer layer, are broken up into a $20 \times 20$ array of discreet single domain magnetic elements. The elements are intended to simulate the magnetic grains in the thin film and have typical dimensions of $50 \mathrm{~nm} \times 50 \mathrm{~nm} \times 2 \mathrm{~nm}$. The elements within each layer are separated by a diffused Ag layer of thickness $\mathrm{G}$, and the elements in different layers are separated by the $\mathrm{Ag}$ layer thickness $t_{\mathrm{Ag}}$. Each element has a magnetic moment of fixed magnitude whose direction is allowed to precess and relax under the influence of an applied magnetic field, local anisotropy field, magnetostatic interactions, and exchange interactions. The magnetostatic interactions are treated exactly using a Green's function formulation for surface magnetic charges. The exchange and anisotropy fields are chosen from appropriate distributions.

Data from a simulation is shown in Fig. 4 for a zero field remnant state. For this simulation there were no exchange interactions between elements and each element had an anisotropy field of $1.6 \mathrm{kA} / \mathrm{m}(20 \mathrm{Oe})$ assigned in a random direction. Finite boundary conditions are applied along the $x$ direction while periodic boundary conditions are applied

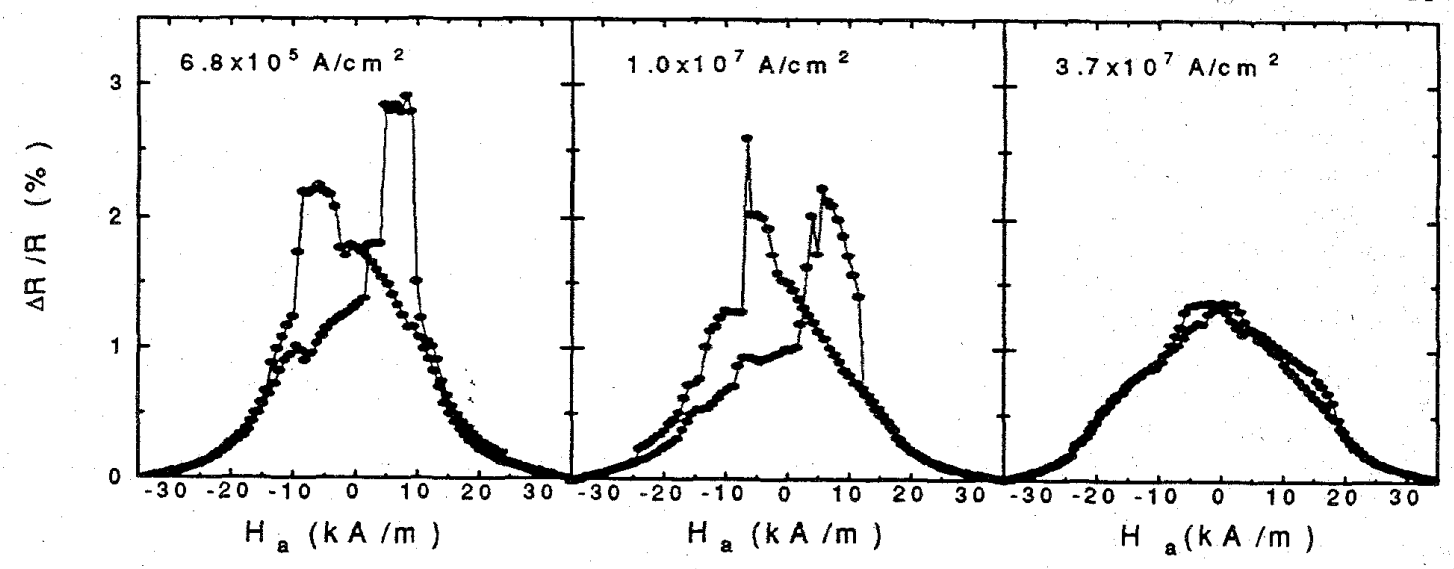

Fig. 2. Magnetoresistive response of a $0.7 \mu \mathrm{m}$ 7-bilayer NiFe/Ag device for different current densities. 


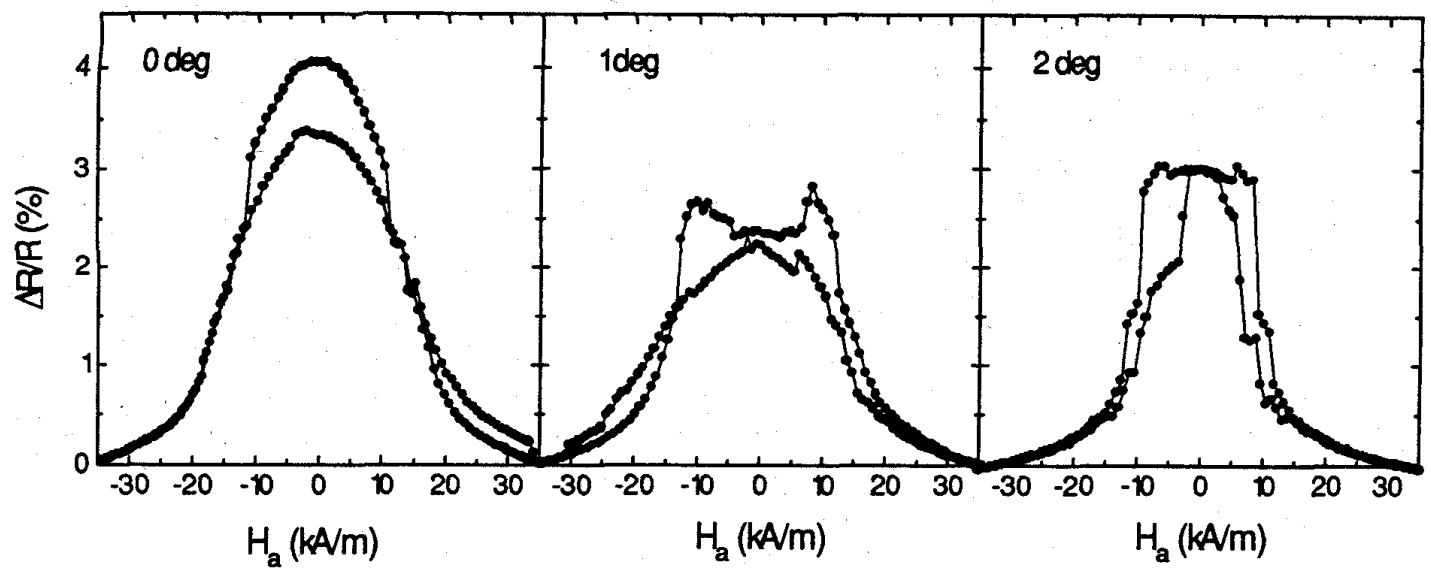

Fig. 3. Magnetoresistive response of $0.7 \mu \mathrm{m}$ 7-bilayer NiFe/Ag device for different field angles. The field angle is measured from the direction perpendicular to the current density and the current density was held constant at $5.0 \times 10^{5} \mathrm{~A} / \mathrm{cm}^{2}$.

along the y direction. This simulates a device of width $1 \mu \mathrm{m}$ and infinite length. The magnetic moments at the edges of the finite dimension are aligned with the device edges. This configuration gives few free charges at the device edges which minimizes the magnetostatic energy. As seen, there is a high level of antiparallel correlation between the top and bottom layers. For this simulation, the degree of interlayer antiparallel correlation in the zero field state was $C=\left\langle\vec{m}_{t} \cdot \vec{m}_{b}\right\rangle=-0.8$ where $m_{t, b}$ are neighboring top and bottom magnetic moments.

The giant magnetoresistance contribution is calculated assuming that each element, $\vec{m}$, has a contribution to its resistance from each of its neighbors $\vec{m}_{n}$ of the form $\delta r\left(\vec{m} \cdot \vec{m}_{n}\right)$. Both interlayer and intralayer neighbors

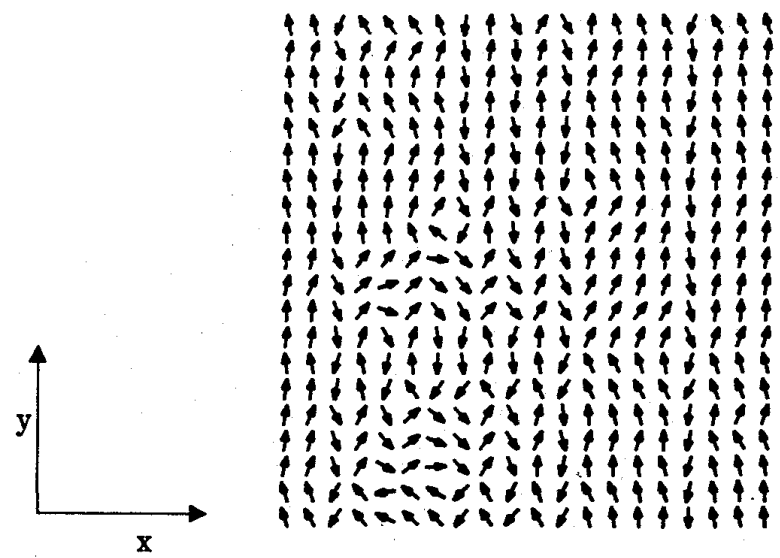

Top contribute but the interlayer contribution to the GMR dominates due to the higher degree of antiparallel correlation and larger common area. The device resistance is then calculated from a resistor network model of the magnetic elements assuming the current flows along the $y$ direction. The magnetoresistance for the simulation in Fig. 4 is shown in Fig. 5. While there is some structure in the magnetoresistive response, to model the large Barkhausen jumps seen in the experimental data one must incorporate some exchange coupling or contact between grains.

Fig. 6 shows the zero field state of a simulation of a 0.5 $\mu \mathrm{m}$ device which includes a ferromagnetic exchange interaction between the elements within each layer. For the simulation shown, a uniform exchange value of $\mathrm{A}=1 \times 10^{-7}$ $\mathrm{erg} / \mathrm{cm}$ was used ( $\sim 10 \%$ of the bulk value). The exchange interaction forces larger clusters of elements to switch coherently leading to large Barkhausen jumps as shown in

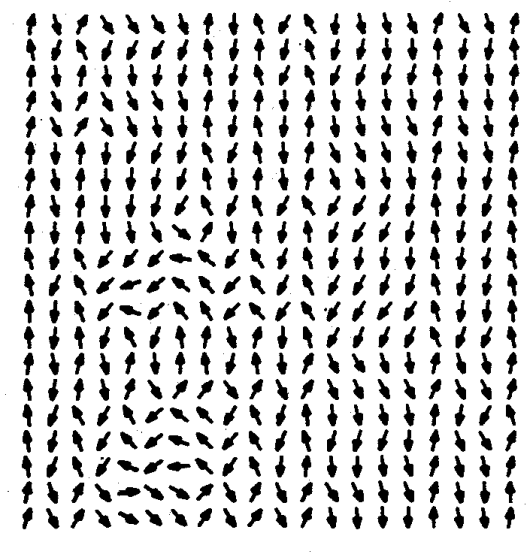

Bottom

Fig. 4. Magnetization of elements in the top and bottom NiFe layers in the zero field state for simulation for which there was no exchange between elements. The spacing between layers was $\mathrm{t}_{\mathrm{Ag}}=4.0 \mathrm{~nm}$, the grain size was $50 \mathrm{~nm} \times 50 \mathrm{~nm} \times 2 \mathrm{~nm}$, the separation between elements due to $\mathrm{Ag}$ diffusion was $\mathrm{G}=1.0 \mathrm{~nm}$, and a randomly oriented anisotropy of magnitude $1.6 \mathrm{kA} / \mathrm{m}(20 \mathrm{Oe})$ was used. 
Fig. 7. The magnitude of the Barkhausen jumps are closer to those observed in real devices. However, to accurately simulate real devices, a distribution of exchange strengths, with both negative and positive values, should be used to account for the variations in the grain separation, $G$, and the oscillatory dependence of the exchange interaction on $\mathrm{G}$.

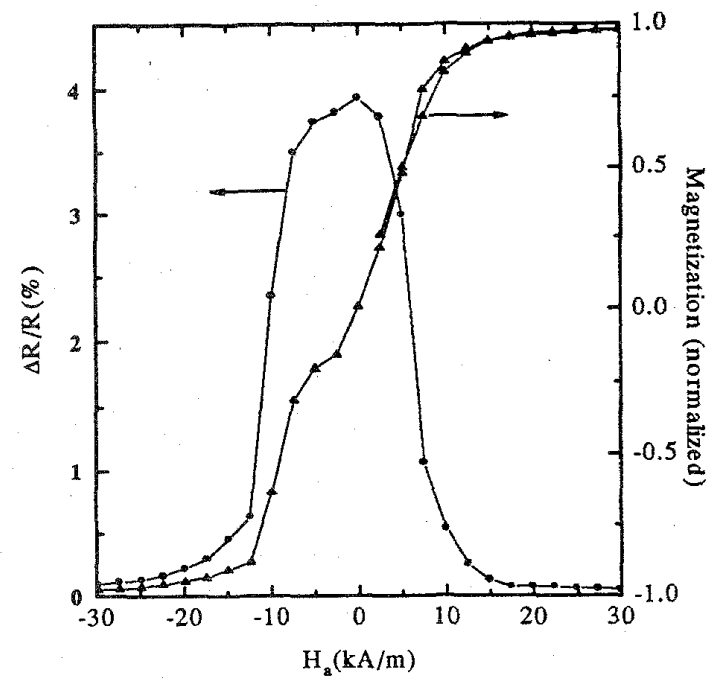

Fig. 5. Magnetoresistive response and magnetization of simulation described in Fig. 4. The field was perpendicular to the current density.

\section{CONCLUSIONS}

We have studied the magnetoresistive response of submicron annealed NiFe/Ag GMR devices both experimentally and with micromagnetic simulations. This system contains a rich domain structure which can lead to large Barkhausen jumps in submicron devices. The detailed domain structure and switching behavior is a sensitive function of magnetic field angle and current density. Large Barkhausen jumps occur when the field angle deviates from

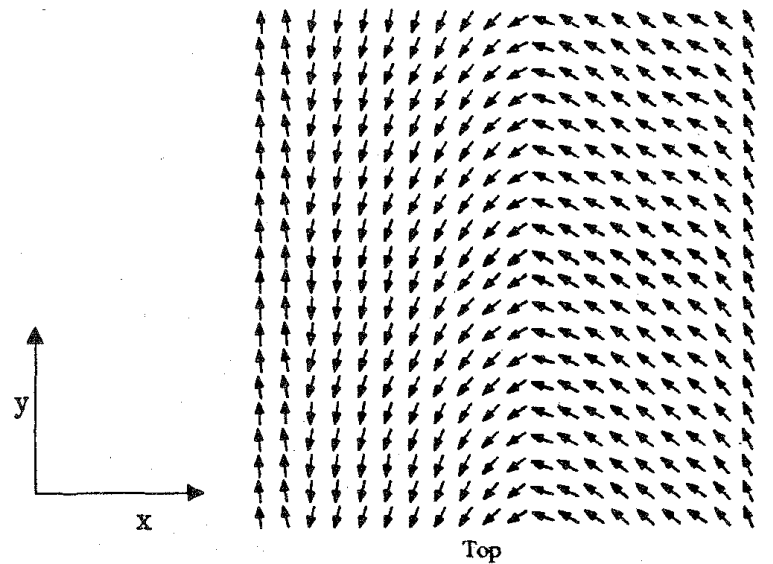

the direction perpendicular to the current density, while going to high current density smoothes out the magnetoresistive response.

\section{REFERENCES}

[1] R.W. Cross, S. E. Russek, S. C. Sanders, M.R. Parker, J.A. Bamard, and A. A. Hossain, "Size and Self-Field Effects in Giant Magnetoresistive Thin-Film Devices," IEEE Trans. Mag. 30, pp. 3825-3827, 1994.

[2] T. L. Hylton, K. R. Coffey, M. A. Parker, and J.K. Howand, "Giant magnetoresixtance at low fields in discontinuous $\mathrm{NiFe}-\mathrm{Ag}$ Multilayer Thin Films," Science 261, pp. 1021-1024, 1993.

[3] T. L. Hylton, K. R. Coffey, M. A. Parker, and J.K. Howard, "Low field giant magnetoresistance in discontinuous magnetic multilayers," J. Appl. Phys. 75, pp. 7058-7060, 1994.

[4] Y. K Kim and S. C. Sanders, "Magnetostriction and giant magnetoresistance in annealed NiFe/Ag multilayers," Appl. Phys. Lett. 66, pp. 1009-1011, 1995.

[5] J. C. Slonczewski, "Magnetostatic mechanism for field sensitivity of magnetoresistance in discontinuous magnetic multilayers," J. Magn. Mat., 129, pp L123-L128, 1994.

[6] J. O. Oti, S. E. Russek, S. C. Sanders, and R. W. Cross, "Models of granular giant magnetoresistance multilayer thin films," in press:

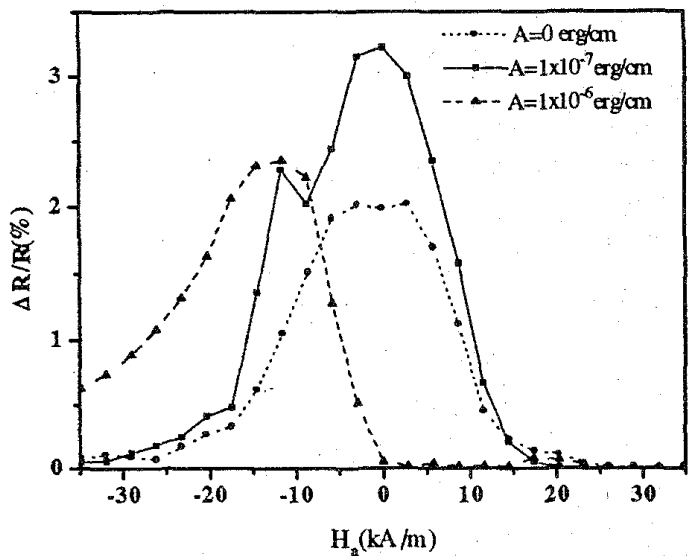

Fig. 7. Magnetoresistive response of simulation described in Fig. 6 for different values of exchange coupling. For the intermediate exchange strength Barkhausen jumps can be observed. For the highest exchange strength there is a large hysteresis- only one branch is shown. The field was perpendicular to the current density.



Fig. 6. Magnetization of elements in the top and bottom NiFe layers in the zero field state for simulation for which the exchange between elements was $1 \times 10^{-7}$ erg $/ \mathrm{cm}$. The spacing between layers was $t_{A B}=4.0 \mathrm{~nm}$, the grain size was $25 \mathrm{~nm} \times 25 \mathrm{~nm} \times 2 \mathrm{~nm}$, the separation between elements due to $A g$ diffusion was $G=$ $1.5 \mathrm{~nm}$, and a randomly oriented anisotropy of magnitude $1.6 \mathrm{kA} / \mathrm{m}(20 \mathrm{Oe})$ was used. 